\title{
Flüssigkeitsstrahllöten - ein Verfahren zur Herstellung von Verbindungen an extrem dünnen Bauteilen
}

\author{
Wspomagane laserowo spajanie strumieniem ciekłego lutu \\ - technologia łączenia ekstremalnie cienkich elementów
}

\section{Zusammenfassung}

Das Automobil der Zukunft muss die wach-senden gesellschaftlichen Erwartungen im Hinblick auf Energieeffizienz, Kraftstoffver-brauch, Klimaschutz, Sicherheit, Komfort und Nachhaltigkeit erfüllen. Dem Leichtbau kommt dabei eine besondere Rolle zu. Neu-ere Entwicklungen gestatten es, dem Endan-wender - der Mobilität produzierenden Indu-strie - bereits mit vorgefertigten Leichtbau-verbundwerkstoffen, den Stahl-Kunststoff-Stahl-Verbundwerkstoffen, zu arbeiten. Diese neuen Stahl-Kunststoff-Stahl-Verbundwerkstoffe intelligent zu nutzen, erfordert geeignete Fügetechnologien. Das tragfähige Verbinden von StahlKunststoff-Stahl-Verbundwerkstoffen unter Beibehaltung des Multimaterialkonzeptes wird zum zentralen Baustein der Realisierung von Leichtbau. Ziel des Beitrages ist es, eine neuartige, effiziente Fügetechnologie zur Herstellung von Karosseriekomponenten mit Stahl-Kunststoff-StahlVerbundwerkstoffen und konventionellen Stählen durch den Einsatz thermischer Energie in Form eines metallischen Flüssigkeitsstrahles vorzustellen.

Keywords: Flüssigkeitsstrahllöten; Stahl-Kunststoff-StahlVerbundwerkstoffe; Laseraktivierung

\section{Streszczenie}

Motoryzacja przyszłości musi sprostać rosnącym społecznym oczekiwaniom związanym z efektywnym wykorzystaniem energii, zużyciem paliwa, ochroną środowiska, bezpieczeństwem, komfortem i trwałością. Lekkie konstrukcje pojazdów odgrywają przy tym szczególną rolę. Najnowsze rozwiązania technologiczne pozwalają użytkownikowi - w tym wypadku branży motoryzacyjnej - pracować z odpowiednio przygotowanymi lekkimi kompozytami na bazie stali i tworzywa sztucznego. Inteligentne zastosowanie tych kompozytów typu sandwich wymaga odpowiedniej technologii łączenia. Uzyskanie połączeń kompozytów, które spełniają warunki nośności, przy uwzględnieniu wielomateriałowej struktury pojazdu będzie głównym aspektem realizacji lekkich konstrukcji w motoryzacji. Celem artykułu jest przedstawienie nowej, efektywnej technologii wykonywania połączeń elementów karoserii z blach kompozytowych i konwencjonalnych z wykorzystaniem energii cieplnej strumienia ciekłego metalu i aktywacji laserowej.

Słowa kluczowe: spajanie strumieniem ciekłego lutu; materiał kompozytowy stal - tworzywo sztuczne - stal; aktywacja laserowa

\section{Zielstellung/Motivation}

Für die Herstellung von Verbindungen an dünnen Bauteilen wurde eine Vielzahl neuer Fügeverfahren entwickelt. Neben dem Kleben kommen zunehmend mechanische Fügeverfahren bzw. Verfahrenskombinationen zur Anwendung. Weniger geeignet sind derartige Verbindungen jedoch im sichtbaren Bereich der Außenhaut von Fahrzeugen. Zusätzliche Maßnahmen werden notwendig, um die Verbindungsstelle an die notwendigen und ästhetischen Ansprüche anzupassen. Zusätzliche Kosten sowie die begrenzte physikalisch-chemischen Beanspruchbarkeit der Verbindung sind Ursachen für Überlegungen und Untersuchungen mit dem Ziel, neuartige effiziente metallische Verbindungen in diesen Bereichen der Fahrzeuge zu schaffen. Ein wesentlicher Schritt, die Leichtbaubestrebungen durch die thermische Fügetechnik zu unterstützen, war die Einführung niedrig schmelzender Zusatzwerkstoffe und ihre Verarbeitung mit konventionellen Verfahren der Schweißtechnik. Der Übergang vom Eisen(Fe)Basis-Zusatzwerkstoff zu einem Kupferbasis(Cu)-Werkstoff führt zu einer Reduzierung der spez. Energie für die Verflüssigung des Zusatzwerkstoffes um 24\%. Der Grundwerkstoff wird nicht bzw. nur begrenzt aufgeschmolzen und es gelingt dadurch, in der Serienfertigung Blechdicken von ca. 0,6 mm

Prof. Dr.-Ing. Ralf Winkelmann, M. Eng. Andrè Kilian, Dipl.-Ing. Daniel Serafinski - BTU Cottbus-Senftenberg, Fertigungstechnik/Tribologie.

Autor korespondencyjny/Corresponding author. ralf.winkelmann@b-tu.de 
zu verbinden. Eine Zerstörung der häufig zum Zwecke des Korrosionsschutzes auf Blechen des Karosseriebaus aufgebrachten Zink-Beschichtung kann durch die Anwendung von Cu-Loten nicht verhindert werden. Die Verarbeitung von Cu-Loten in der Serienfertigung von Fahrzeugen erfolgt sowohl mittels Strahltechnik als auch mit Lichtbögen.

Die Anwendung hochfester Stähle und die Kombinationen von Werkstoffen bedingen weiterreichende Anforderungen an die Fügetechnik. Eine neuere Entwicklung sind die Stahl-Kunststoff-Stahl-Verbundwerkstoffe. Sie bieten ein hohes Leichtbaupotenzial und werden infolge der großtechnischen Herstellung auch als wirtschaftlich attraktiv beurteilt. Sie thermisch zu fügen, ist eine zu lösende Aufgabe. Es sind Verbindungen an dünnen Bauteilen (Einzelblechdicken $\leq 0,3 \mathrm{~mm}$ ) unter Beachtung einer thermisch begrenzten Beanspruchbarkeit des Stahl-Kunststoff-Verbundes zu fügen. Wird nun noch das Ziel verfolgt, einen metallischen Verbund zu schaffen, werden Zusatzwerkstoffe mit geringer Schmelzenergie die Technologieentwicklung zielführend unterstützen. Es bieten sich Werkstoffe auf der Basis von Zink (Zn) oder auch Zinn (Sn) an. Gegenüber den Fe-Basis-Zusatzwerkstoffen wird die Energie zur Verflüssigung um 74\% (Zn) bzw. um 90\% (Sn) reduziert. Um derartige Werkstoffe schmelzmetallurgisch mit dem Ziel der Herstellung von Verbindungen an dünnen Bauteilen bei begrenzter thermischer Belastung des Grundwerkstoffes zu verarbeiten, bedarf es geräte- und verfahrenstechnischer Entwicklungen. Randbedingungen derartiger Entwicklungen sind: Fügegeschwindigkeiten bis $4 \mathrm{~m} / \mathrm{min}$; gleichmäßige Geometrie und damit geringe Nacharbeit; keine Delamination des Stahl-Kunststoff-Verbundes; konkave Nahtgeometrie an Bördelstößen und Zugfestigkeiten $>100 \mathrm{~N} / \mathrm{mm}^{2}$; angestrebte Verbindungsfestigkeiten > Verbindungsfestigkeit geklebter Verbindungen.

\section{Verfahrensauswahl / Randbedingungen}

Moderne vorgefertigte Leichtbauverbundwerkstoffe, die Stahl-Kunststoff-Stahl-Verbundwerkstoffe $[1,2]$ bestehen aus sehr dünnen Deckblechen mit Stärken von 0,2 bis $0,25 \mathrm{~mm}$, zwischen denen sich eine Kernschicht aus einem Kunststoff variabler Dicke befindet. Diese Werkstoffe sind nur unwesentlich schwerer (ca. 10\%; [2]) als Aluminium, weisen jedoch eine deutlich höhere Biegesteifigkeit $(E * I)$ auf. Von großer Bedeutung für die Umformtechnik sind die kleinen einstellbaren Radien, welche deutlich unter denen liegen, die beim Umformen von Aluminium darstellbar sind. Um diese Werkstoffe im Außenhautbereich einer Karosserie mit sich selbst bzw. mit konventionellen Stählen thermisch zu verbinden, bedarf es der Entwicklung einer neuen Fügetechnologie - da es auch mit modernsten Geräten der Fügetechnik nicht gelungen ist, Verbindungen ohne Delamination des Stahl-Kunststoff-Verbundes herzustellen. Die Wirkung modernster, in der thermischen Fügetechnik angewandter, geregelter, extrem kalter Lichtbögen $([3,4])$ führt zur Zerstörung des sich zwischen den beiden 0,2 bis $0,25 \mathrm{~mm}$ dicken Stahlblechen befindenden Kunststoffs bzw. zu dessen Ablösung vom Stahlblech (Fig. 1). Allein die Erwärmung als Folge des Stromflusses (60 A) vom Lichtbogenfußpunkt zur Masseklemme führt in der 0,2 $\mathrm{mm}$ dicken Blechlagen des Stahl-Kunststoff-Stahl-Verbundteiles zu einer Erhöhung der Temperatur um ca. $220 \mathrm{~K}$. Temperaturmessungen während des Fügens dieser Stahl-Kunststoff-Stahl-Verbundwerkstoffe unter Nutzung geregelter Kurzlichtbogentechnik (KLB) bei Anwendung sehr niedrig schmelzender Zusatzwerkstoffe auf Sn-Basis ergeben Werte an der Bauteiloberfläche von ca. $400{ }^{\circ} \mathrm{C}$. Delaminationen können unter diesen Bedingungen nicht verhindert werden. Anzustreben sind Temperaturen unter $250{ }^{\circ} \mathrm{C}$ im Übergangsbereich vom Stahl zum Kunststoff.

In Figure 1 ist eine mittels geregelter KLB gefügte Probe nach dem Zugversuch dargestellt. Deutlich erkennbar ist die Zerstörung der Verbindung Stahl-Kunststoff infolge der thermischen Wirkung des Lichtbogens. Diese Verbindung wurde mit einer Streckenenergie von $620 \mathrm{~J} / \mathrm{cm}$ bei einer Schweißgeschwindigkeit von $25 \mathrm{~cm} / \mathrm{min}$ hergestellt. Um den Lichtbogen stabil zu halten, war ein Positivanteil von 65 $\%$ erforderlich. Die Verbindungsfestigkeit betrug $125 \mathrm{~N} / \mathrm{mm}^{2}$ - bezogen auf die Gesamtdicke (1 mm) des Stahl-KunststoffStahl-Verbundwerkstoffes. Die Zugbeanspruchung führt zu einer deutlichen Deformation der delaminierten Zone und im Folgenden wird erst die Lötverbindung im Übergangsbereich zur Beschichtung bzw. zum Grundwerkstoff zerstört. Um derart hohe Festigkeiten zu erzielen, wurde ein Zn-BasisZusatzwerkstoff (ZnAl4) benutzt. Auch die Anwendung von Lasern ergab ähnliche Ergebnisse. In Figure 2 sind diese beispielhaft verdeutlicht.

Mit diesen Versuchen ist der Nachweis dafür erbracht, dass die existierenden modernen Geräte der thermischen Fügetechnik unter Nutzung von $\mathrm{Cu}$ und $\mathrm{Zn}$ als Zusatzwerkstoffe $z$. Zt. nicht geeignet sind, diese neuen StahlKunststoff-Stahl-Verbundwerkstoffe thermisch linienförmig zu fügen. Sollen also die Vorteile der thermischen Fügetechnik, wie die Herstellbarkeit dichter, artähnlicher, korrosionsbeständiger, metallischer, linienförmiger Verbindungen hoher mechanischer Beanspruchbarkeit, auch für diese Werkstoffe genutzt werden, ist die Wirkung des Lichtbogens am Grundwerkstoff zu beeinflussen. Dies kann durch die Anwendung nicht übertragener Lichtbögen bzw. die konsequente Trennung zwischen dem Aufschmelzen des Lotzusatzwerkstoffes und der Aktivierung der Verbindungsstelle erreicht werden.

Es sollen die bekannten Vorteile der Lichtbogenlöt- bzw. Laserlötprozesse, wie:

- die problemlose Integration in die Roboterfertigung (Automatisierbarkeit),

- nur einseitige Zugänglichkeit (gegenüber Clinchen oder Stanznieten),

- spalt- und positionstolerant (Erstar-rungsbedingungen) sowie

- keine aufwendigen Vorbehandlung und längeres Fixieren (gegenüber Kleben) erhalten bleiben. Diese Bedingungen werden mit der in Figure 3 dargestellten Anordnung eingehalten.
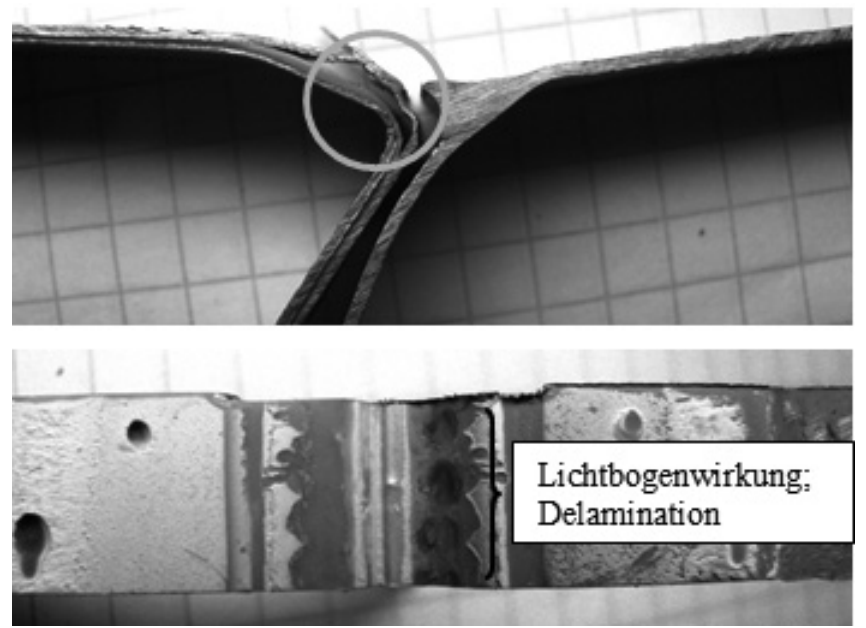

Bild 1. Oben zerstörte Verbindung; unten Lichtbogenwirkung am Verbund

Rys. 1. U góry zniszczone połączenie, na dole efekt oddziaływania łuku elektrycznego w obszarze połączenia 


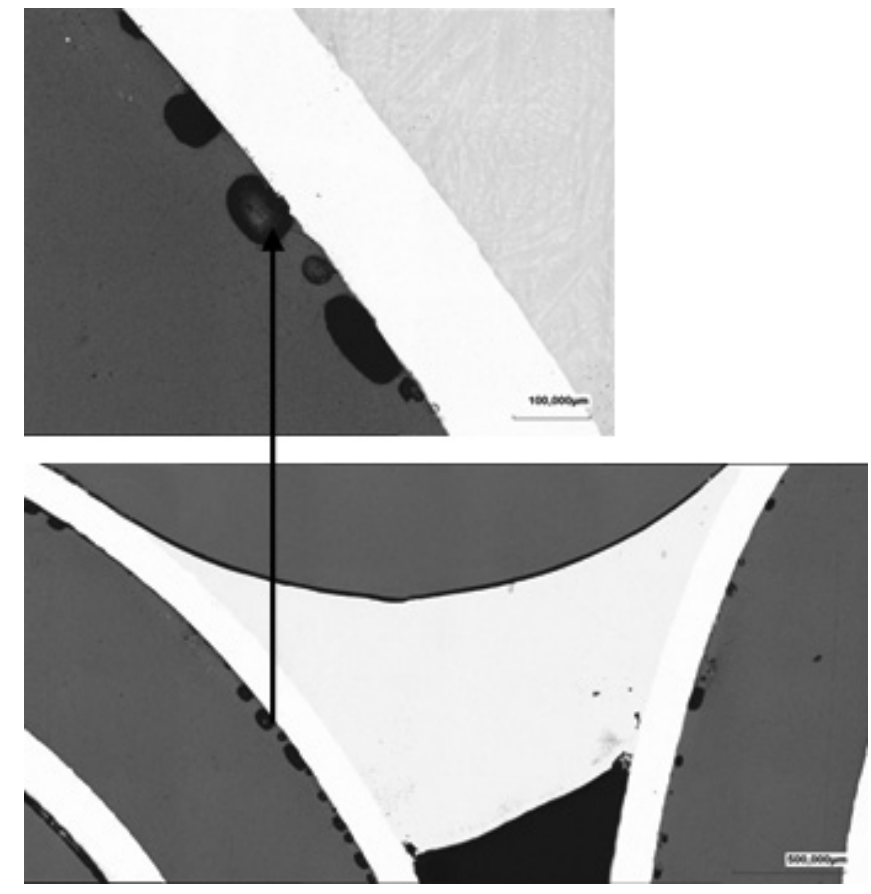

Bild 2. Laserlötung; CuSi3, Delamination

Rys. 2. Lutowanie laserowe, rozwarstwienie
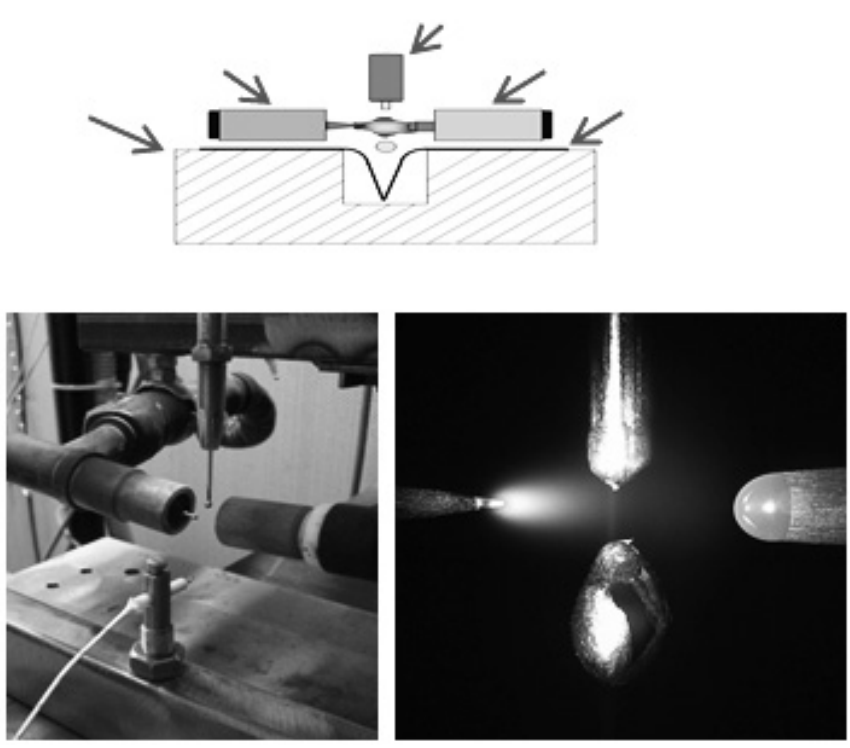

Bild 3. Oben Schema, unten links Versuchsanordnung; unten rechts Tropfenablösung

Rys. 3. U góry schemat, na dole z lewej stanowisko badawcze, z prawej odłączenie kropli lutu

Mit dieser Konfiguration ist es gelungen, Verbindungen herzustellen, die den Anforderungen schon sehr nahe kommen.

In Figure 4 ist eine Bördelnaht dargestellt. Ein 0,7 mm dickes verzinktes Blech der Stahlsorte DX56D + Z100 (1.0963) und ein Stahl-Kunststoff-Stahl-Verbundblech $(0,2+0,6+$ $0,25 \mathrm{~mm}$ ) wurden mit dem Zusatzwerkstoff SnCu3 bei einer Geschwindigkeit von $32 \mathrm{~cm} / \mathrm{min}$ reproduzierbar gefügt. Die bei konventionellen Lötungen vorhandenen Delaminationen konnten nahezu vollständig vermieden werden (Fig. 5). Bei dieser Konstellation der Energiebereitstellung werden

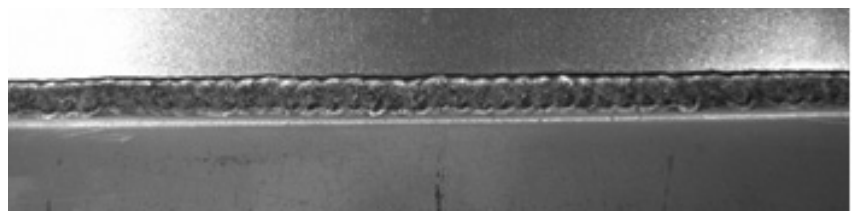

Bild 4. Bördelnaht

Rys. 4. Złącze przylgowe die Aufschmelzung des Zusatzwerkstoffes und die Oberflächenaktivierung mit einer Energiequelle bewerkstelligt. Dadurch ist der Prozess schwierig an hohe Abschmelzleistungen bzw. hohe Fügegeschwindigkeiten bei gleichzeitiger Prozeßstabiltät anpassbar. Eine Trennung zwischen der für die Aufschmelzung bereitzustellenden Energie und der Energie für die Oberflächenaktivierung ist anzustreben.

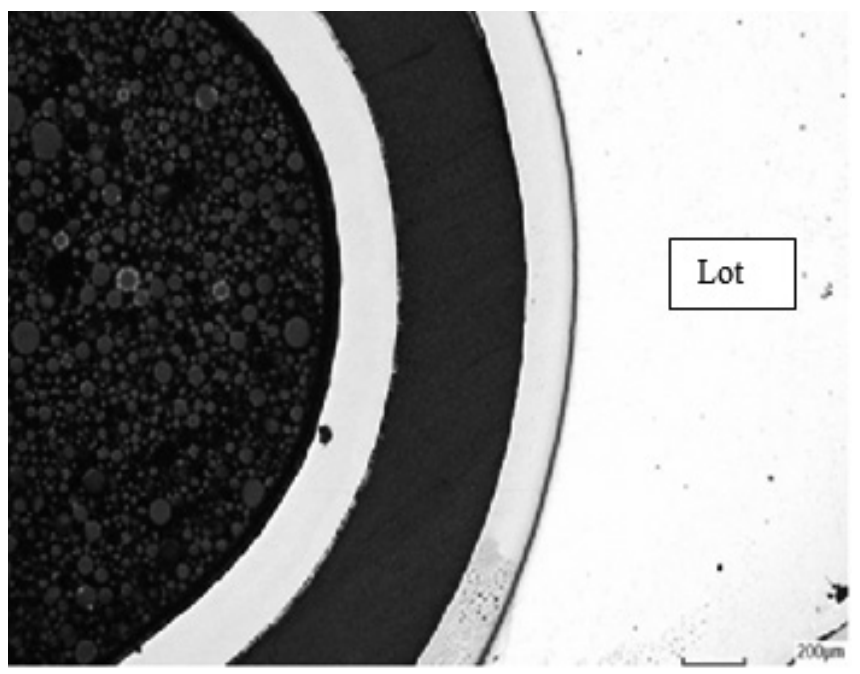

Bild 5. Lötung ohne Delamination

Rys. 5. Połączenie lutowane bez rozwarstwienia

Sichere Verbindungen (mittlere Zugfestigkeit beträgt 110 $\mathrm{N} / \mathrm{mm}^{2}$ ) konnten bei dieser Konstellation mit I = 52,0 A und $\mathrm{U}=15,1 \mathrm{~V}$ hergestellt werden.

\section{Flüssigkeitsstrahllöten}

Um Verbindungen mittels Löten herzustellen, müssen Diffusionsprozesse initiiert werden. Diese sind entsprechend dem 1. Fick`schen Gesetz (1) abhängig von der Temperatur $(T)$, dem Druck (p) und dem chemischen Potenzial (Konzentration c). Es besteht eine Proportionalität zwischen der Teilchenstromdichte $\vec{J}_{D}$ und dem Konzentrationsgradienten $\partial c / \partial x$. Diese wird durch den Diffusionskoeffizienten $D$ beschrieben.

$$
\vec{J}_{D}=\left(-D \frac{\partial c}{\partial x}\right)_{T ; p}
$$

Da der Druck weitestgehend konstant angenommen werden kann, stehen nur die Temperatur und der Zusatzwerkstoff als Pa-rameter zu Verfügung. Um eine möglichst geringe Energie über den Tropfen in die Verbindung einzubringen, wurden die Untersuchungen mit Sn-Basis-Loten durchgeführt. Die Reaktivität $\mathrm{Sn-Zn}$ ist sehr hoch. Sie kann jedoch aufgrund der Existenz von Hydroxyd-schichten an der Oberfläche der Zn-Beschichtung nicht von der Sn-Schmelze allein ausgelöst werden. In Figure 6 ist dies durch das Zuschalten einer Aktivierung mittels Laser verdeutlicht.

Der Diffusionskoeffizient D kann entsprechend der Arrhenius-Gleichung (2) bestimmt werden.

$$
D=D_{0} \exp ^{-\frac{\Delta E}{R * T}}
$$

Mit $\Delta E$ ist die Aktivierungsenthalpie, mit $\mathrm{R}$ die molare Gaskonstante und mit $D_{0}$ ist eine für das betrachtete System konstante Größe definiert. Es kann in grober Näherung angenommen werden, dass die Mindesttemperatur an der 


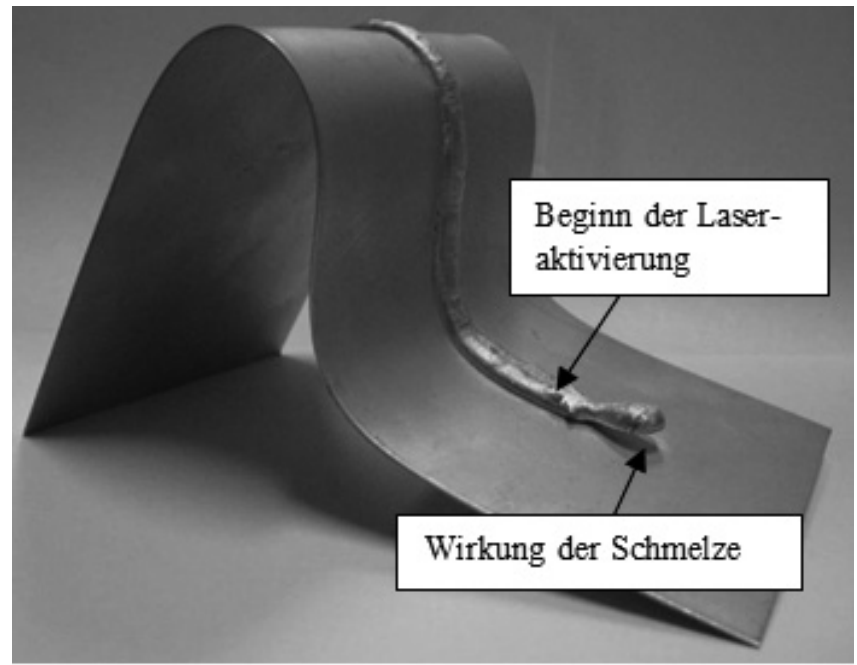

Bild 6. Oberflächenaktivierung

Rys. 6. Laserowa aktywacja powierzchni

Verbindungsstelle zur Auslösung technisch sinnvoller Diffusionsprozesse entsprechend der Beziehung (3) berechnet werden kann.

$$
T \geq 0,4 \cdot T_{s}
$$

Somit muss die Temperatur einen Wert von $T \geq 0,4 \cdot 420=$ $=168^{\circ} \mathrm{C}$ annehmen, um Verbindungen zur Zn-Beschichtung zu ermöglichen. Da die maximale thermische Belastung des Stahl-Kunststoff-Verbundes bei $250{ }^{\circ} \mathrm{C}$ liegt, existiert ein Arbeitsbereich von ca. $80 \mathrm{~K}$. Diese geringe Differenz einzuhalten, ist eine technologische Herausforderung. Eine Lösung hierfür ist das Flüssigkeitsstrahllöten. Ein Behälter, in welchem sich der Lotzusatzwerkstoff befindet, wird z. B. induktiv erwärmt. Nach dem Erschmelzen des Lotes und dem Öffnen des Ventiles verlässt diese als Flüssigkeitsstrahl den Behälter durch eine Düse. In Figure 7 ist der innovative Behälter dargestellt. Der Zusatzwerkstoff wird als Draht zugeführt. Um mögliche Reaktionen des Lotes mit der Atmosphäre zu reduzieren, bietet es sich an, mit Schutzgas zu arbeiten. Es wird Argon (Ar) oder auch Stickstoff (N2) benutzt. Die Temperatur der Schmelze wird indirekt am Rand des Behälters gemessen. Das Messsignal wird für die Steuerung des Generators benutzt. Somit gelingt es, die Schmelze im Behälter auf einer definierten Temperatur zu halten. Die Zuführung des kalten Lotes erfolgt entsprechend dem Abfluss. Es wird ein definierter Füllstand eingestellt - wodurch sich bei konstantem Düsendurchmesser ein kontinuierlicher Volumenstrom ergibt. Damit ist eine Energiequelle definiert. Entsprechend den durchgeführten Berechnungen wird bei Anwendung von Sn-Basis-Loten in die Verbindung eine Streckenenergie von ca. $145 \mathrm{~J} / \mathrm{cm}$ unter Vernachlässigung von Verlusten eingetragen. Damit konnte die Streckenenergie gegenüber den bei Anwendung modernster Lichtbogentechnik ermittelten Werten um den Faktor 4,3 reduziert werden.

Werden Flussmittel für die Aktivierung der Oberfläche benutzt, ist dieser Energieeintrag ein Minimum. Flussmittel werden bei der Herstellung von Fahrzeugen selten benutzt, so dass eine andere Möglichkeit der Aktivierung zu erarbeiten war. Eine Aktivierung der Oberfläche durch die Wirkung eines übertragenen Lichtbogens, welcher durchaus sehr positive Effekte bewirkt, war keine Lösung. Der Lichtbogen war bei den angestrebten hohen Geschwindigkeiten von $4 \mathrm{~m} / \mathrm{min}$ nicht stabilisierbar. Somit kam die Laserstrahlung zur Anwendung. Als sehr gut geeignet erweist sich aufgrund des relativ großen Fokus der Diodenlaser. Zu beachten ist bei dieser Einschätzung noch die ungleiche Wärmeführung in den Grundwerkstoffen bei der Verbindung zwischen dem massiven DX56D + Z100 (1.0963) und dem Stahl-Kunststoff-

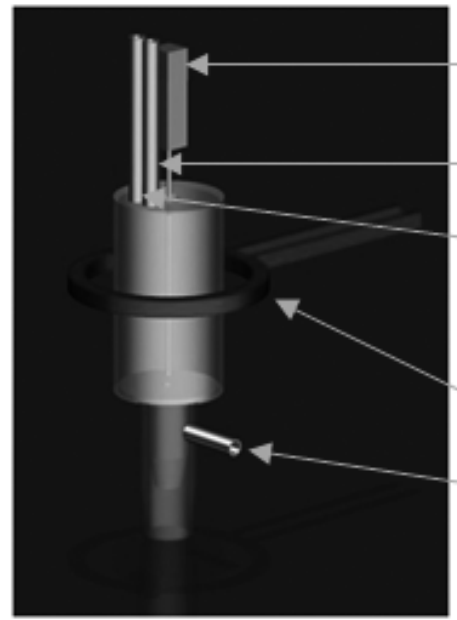

Elektrom agnetisches

Verschlusssystem

Drahtzuführung

Ga szuführungfürkonti-

nuierlichen Schm elzaus-

fluss

Induktionsschleife

Schutzga sanschluss

Schutzga sdiuse

Bild 7. Schmelzbehälter

Rys. 7. Zbiornik do topienia lutu

Stahl-Verbundblech. Der Laserstrahl war entsprechend zu positionieren.

\section{Ergebnisse}

Zum Erreichen der Zielstellung eine geringe Delamination zu erwirken, war es erforderlich, die Delamination selbst zu quantifizieren. Es wurden Blindraupen hergestellt und Probekörper entsprechend Figure 8 präpariert. Bis ca. $10 \mathrm{~mm}$ vor der Blindraupe wurde der Kunststoffkern zwischen den beiden Stahlteilen entfernt. Diese Proben wurden in die Zugmaschine eingespannt, um die Stahlteile von dem Kunststoffkern abzuschälen. Eine Delamina-tion (Fig. 9) ist deutlich erkennbar und messtechnisch gut zu erfassen. Sie wird als Delaminationslänge angegeben.
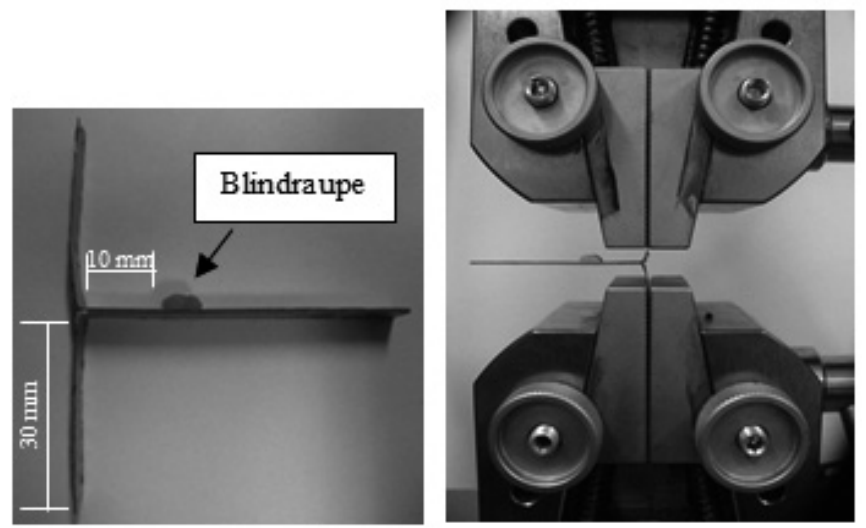

Bild 8. Links Schälzugprobe; rechts Schälzugversuch

Rys. 8. Z lewej próbka do testu rozdzierania, z prawej próba rozdzierania

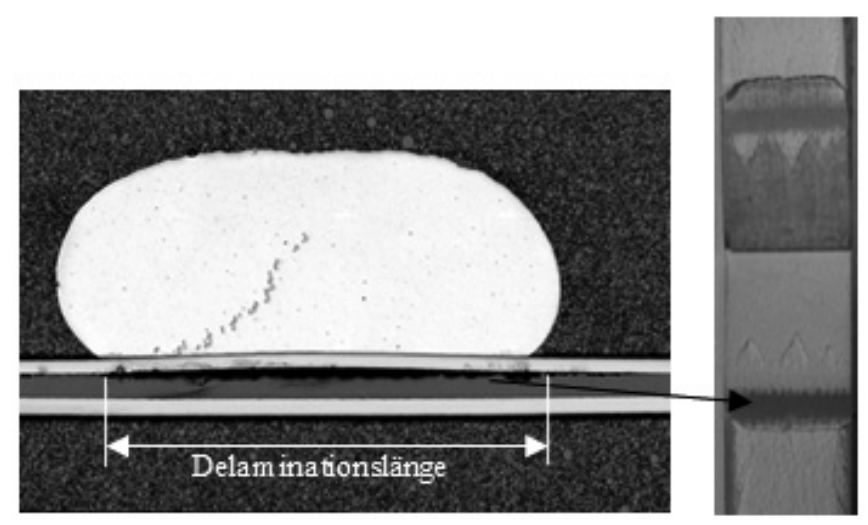

Bild 9. Delamination

Rys. 9. Rozwarstwienie 
Die Ergebnisse der Untersuchungen zu Blindraupen mittels Flussmittelaktivierungen ergaben den in Figure 10 verdeutlichten Zusammenhang. Unter einer Streckenergie von $145 \mathrm{~J} / \mathrm{cm}$ war keine Delamination mehr zu registrieren. Es wurden Prozessgeschwindigkeiten bis $4,1 \mathrm{~m} / \mathrm{min}$ eingestellt.

Bei den Untersuchungen mit Laseraktivierung und identischer Geschwindigkeit konnten keine delaminationsfreien Proben hergestellt werden.

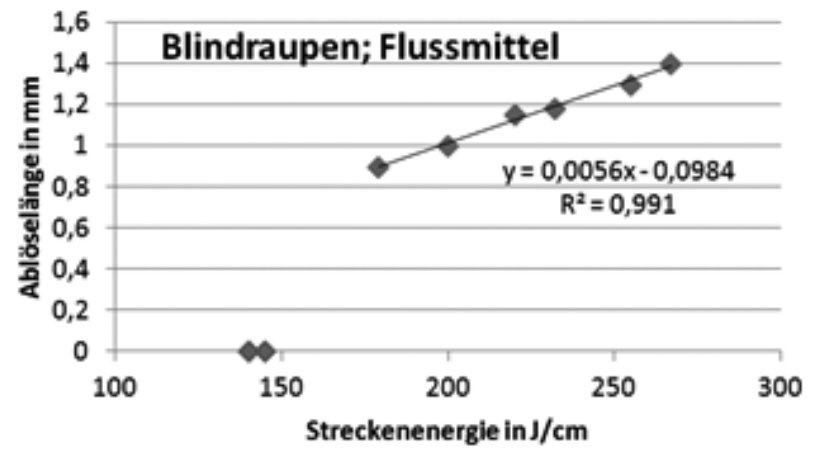

Bild 10. Blindraupen

Rys. 10. Ściegi „ślepe”

Ein geeignetes Zusammenwirken zwischen dem Flüssigkeitsstrahl und der Aktivierung ist in Figure 11 dargestellt. Der Laser muss in Lötrichtung vor dem Flüssigkeitsstrahl wirken. Zu beachten ist die Ausbreitung des Lotes an der Verbindungsfläche. Als sinnvoll erwies sich ein Abstand zwischen Flüssigkeitsstrahl und Laserwirkung von 0,5 mm. Der Laser übernimmt zusätzlich die Aufgabe der Erwärmung der zu fügenden Bauteile, so dass deren Wärmeleitung zu berücksichtigen ist.

Unter diesen Bedingungen konnten bei Laserleistungen von $1,8 \mathrm{~kW}$, einem Fokus von $4,7 \mathrm{~mm}$ und einer gesamten Streckenenergie von ca. $230 \mathrm{~J} / \mathrm{cm}$ delaminationsfreie Verbindungen (Figure 12) hergestellt werden.

Die mittlere Zugfestigkeit der hergestellten Verbindungen liegt bei $106 \mathrm{~N} / \mathrm{mm}^{2}$. Die gemessenen Kräfte wurden bei der Spannungsberechnung, bezogen auf die Probenbreite, multipliziert mit der Gesamtdicke des Stahl-Kunststoff-Stahl-Verbundes. Die Festigkeiten der mittels Flussmittelaktivierung hergestellten Verbindungen liegen in der gleichen Größenordnung.

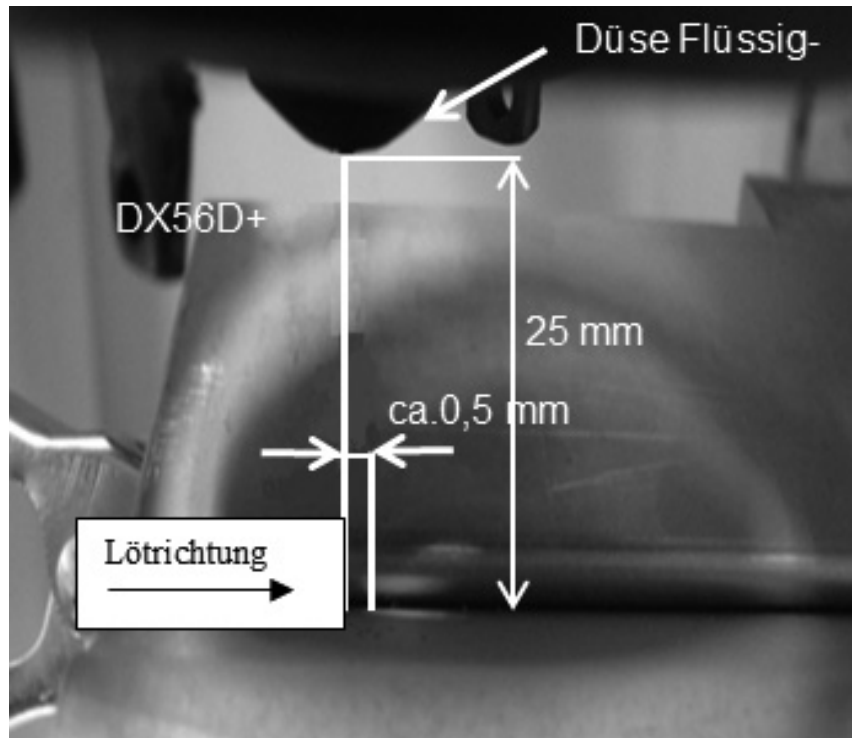

Bild 11. Positionierung Laser-Flüssigkeits-strahl

Rys. 11. Pozycjonowanie strumienia lasera i strugi ciekłego lutu

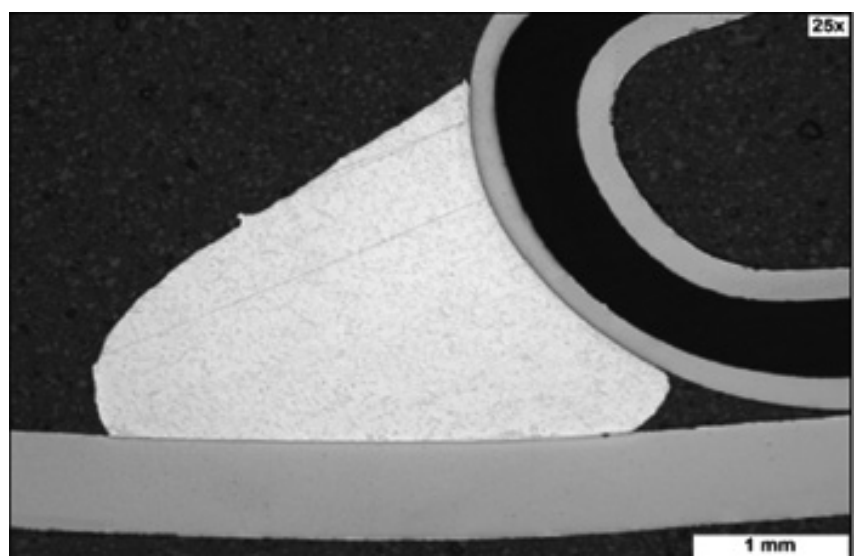

Bild 12. Verbindung DX56D + Z100 (1.0963) zum Stahl-KunststoffStahl-Verbund $(0,2$ / 0,6 / 0,25 mm)

Rys. 12. Połączenie DX56D + Z100 (1.0963) z materiałem kompozytowym stal-tworzywo sztuczne-stal $(0,2$ / 0,6 / 0,25 mm)

\section{Zusammenfassung}

Für die Herstellung metallischer dichter artgleicher Verbindungen zu Stahl-Kunststoff-Stahl-Verbundwerkstoffen mit extrem dünnen Einzelblechlagen wurde das Flüssigkeitsstrahllöten entwickelt. Erforderlich sind eine Verflüssigung des Lotzusatzwerkstoffes, die Aktivierung der Fügestelle sowie die präzise Einhaltung eines Temperaturregimes, um delaminationsfreie Fügungen herzustellen. Die Nutzung der Induktion ist eine Möglichkeit, den Zusatzwerkstoff zu verflüssigen. Um die angestrebten hohen Lötgeschwindigkeiten von ca. $4 \mathrm{~m} / \mathrm{min}$ zu erzielen, bedarf es einer sicheren Aktivierung der Fügestelle. Die Laserstrahlung ist hierfür geeignet. Neben der Zielstellung, bei hohen Fügegeschwindigkeiten delaminationsfreie Verbindungen herzustellen, waren als Randbedingung Verbindungsfestigkeiten definiert, die deutlich über denen von Klebungen liegen. Dies konnte sicher erreicht werden. Weiterhin wurde eine konkave Nahtgeometrie erzielt. Das entwickelte Flüssigkeitsstrahllöten ist geeignet, um Verbindungen zu extrem dünnen Bauteilen herzustellen. Delaminationen des StahlKunststoff-Verbundes sind vermeidbar. Die Festigkeit der Verbindung liegt bei ca. $100 \mathrm{~N} / \mathrm{mm}^{2}$.

Das Forschungsvorhaben wurde u. a. an der Fertigungstechnik/Tribologie der BTU C-S, Senftenberg mit fachlicher Begleitung und mit finanzieller Förderung durch die Forschungsvereinigung Stahlanwendung e.V., Düsseldorf, aus Mitteln der Stiftung Stahlanwendungsforschung, Essen durchgeführt.

\section{Literatur}

[1] ThyssenKrupp (2013): LITECOR ${ }^{\circledR}$ Die neue Art, leicht zu bauen. ThyssenKrupp technikforum 1/2013. Online im Inter net: URL: http://www.thyssenkrupp.com/documents/Publikationen/Techforum/techforum_1_13_ de.pdf, Stand 15.10.2013, S. 48, bis 52.

[2] at2steel (2013): Weltneuheit Leichtblech at2. Online im Internet: URL: http://www.at2steel.com/index.drs4?article_id $=15 \&$ row $=0, \quad$ Stand 15.10.2013
[3] Fronius International (2013): CMT-Schweißen. Online im Internet: URL: http://www.fronius.com/cps/rde/xchg/SID-0AFF0106-760BE388/fronius_international/hs.xsl/79_9399_DEU_HTML.htm, Stand 10.10.2013

[4] EWM Group (2013): coldArc ${ }^{\circledR}$ Die zu- kunftsweisende coldArc ${ }^{\circledR}$ Technologie für Hand- und automatisierte Anwendungen. Online im Internet: URL: http://www.ewm-group.com/de/innovationen/coldarc.html Stand 15.10.2013 